\title{
Urinary Cell-Free miR-99a-5p as a Potential Biomarker for Estrus Detection in Buffalo
}

\author{
Aparna Hebbar, Rajeev Chandel, Payal Rani, Suneel Kumar Onteru and Dheer Singh* \\ Animal Biochemistry Division, Molecular Endocrinology, Functional Genomics and Systems Biology Laboratory, Indian \\ Council of Agricultural Research -National Dairy Research Institute, Karnal, India
}

Accurate estrus detection method is the need of the hour to improve reproductive efficiency of buffaloes in dairy industry, as the currently available estrus detection methods/tools lack high sensitivity and specificity. Recently, circulating miRNAs have been shown as non-invasive biomarkers by various studies. Hence, in order to evaluate their potential as estrus biomarkers, the objective of this study was to identify and compare the levels of 10 hormone-responsive miRNAs in the urine collected at proestrus (PE), estrus (E), and diestrus (DE) phases of buffaloes ( $n$ = 3) pertaining to a discovery sample. Among 10 urinary miRNAs, the levels of bta-mir-99a-5p (E/PE 0.5-fold, $P<0.05$; DE/PE 1.9-fold), bta-miR-125b (E/PE 0.5fold; DE/PE 0.7-fold), bta-mir-145 (E/PE 1.5-fold; DE/PE 0.7-fold), bta-mir-210 (E/PE 1.2-fold, DE/PE 0.7-fold), mir-21 (E/PE 1.5-fold, DE/PE 2-fold), and bta-mir-191 (E/PE

OPEN ACCESS

Edited by:

Cristina Alicia Martinez,

Linköping University, Sweden

Reviewed by:

Archunan Govindaraju,

Bharathidasan University, India Mohammad Abdulkader Akbarsha,

National College, Tiruchirappalli, India

*Correspondence:

Dheer Singh

drdheer.singh@gmail.com

Specialty section:

This article was submitted to Animal Reproduction -

Theriogenology,

a section of the journal

Frontiers in Veterinary Science

Received: 19 December 2020

Accepted: 26 February 2021

Published: 17 May 2021

Citation:

Hebbar A, Chandel R, Rani P,

Onteru SK and Singh D (2021) Urinary

Cell-Free miR-99a-5p as a Potential

Biomarker for Estrus Detection in

Buffalo. Front. Vet. Sci. 8:643910.

doi: 10.3389/fvets.2021.643910 1.3-fold; DE/PE 0.8-fold) were found to be altered during different phases of buffalo estrous cycle. In contrast, bta-mir-126-3p, bta-let-7f, bta-mir-16b, and bta-mir-378 were undetected in buffalo urine. Furthermore, a validation study in an independent group of 25 buffalo heifers showed the increased levels of urinary bta-mir-99a-5p during the DE (3.92-fold; $P<0.0001)$ phase as compared to the E phase. Receiver operating characteristic curve analyses also revealed the ability of urinary miR-99a-5p in distinguishing the $\mathrm{E}$ from the $\mathrm{DE}$ phase (area under the curve of $0.6464 ; P<0.08$ ). In silico analysis further showed an enrichment of miR-99a-5p putative targets in various ovarian signaling pathways, including androgen/estrogen/progesterone biosynthesis and apoptosis signaling, implicating the role of miR-99a-5p in ovarian physiology. In conclusion, significantly lower levels of bta-mir-99a-5p at the $E$ phase than the DE phase in buffalo urine indicate its biomarker potential, which needs to be further explored in a large cohort in the future studies.

Keywords: estrus, buffalo, bta-miR-99a-5p, urine, cell free miRNA, qRT-PCR

\section{INTRODUCTION}

Dairy farming is one of the most significant parts of the agriculture sector in India. Buffaloes are usually found in Southeast Asia and the Mediterranean region (1). They are preferred over other farm animals in India for their higher financial returns via milk and meat, better efficiency in utilizing low-quality feed, and resistance to tropical diseases $(1,2)$. However, poor reproductive efficiency of buffaloes is one of the major limitations hindering their maximum production potential. Several factors lead to poor reproductive performance of buffaloes, including delayed puberty, silent 
heat, variation in calving interval, and low conception rate $(1,3)$, but silent estrus is the major cause of concern (3). In addition, because of lack of an accurate estrus detection method, success of artificial insemination (AI) is limited in buffaloes (4). Generally, estrus is determined by various methods, including hormonal estimation, observation of visual and behavioral signs, gynecoclinical examination, record keeping $(5,6)$, and various devices (7), but their sensitivity and specificity vary in detecting estrus (8). As per an estimate, $\sim 50 \%$ of ovulations remain unnoticed in dairy industry due to diminished estrus behavior (9). Hence, ineffective estrus detection in dairy animals ultimately creates financial loss to the farmers (7). Thus, an accurate estrus detection in buffaloes is essential for effective reproductive management via successful conception, which is usually achieved by carrying out AI generally during ovulation that occurs $\sim 10$ $12 \mathrm{~h}$ after the end of an estrus (E) phase in cattle and buffaloes (10). Therefore, there is an urgent requirement to develop a highly sensitive and specific estrus detection method for buffaloes to increase the AI success rate.

MiRNAs are small non-coding RNAs that mainly regulate gene expression at posttranscriptional level. Literature survey showed a cyclic expression of miRNAs in bovine ovarian tissues during an estrous cycle $(11,12)$, suggesting their specific role in different phases of an estrous cycle. Moreover, miRNAs were also reported to be stably present in extracellular environment, either inside extracellular vesicles such as exosomes or as miRNA protein or miRNA lipoprotein complexes (13-15). Evidences showed the presence of tissue-specific miRNAs in circulation at quantifiable levels (16), indicating their use as a biomarker. For example, higher levels of urinary miR-210-3p in cancer patients were significantly reduced in a disease-free stage (17), demonstrating the tumor as their source of origin. Similarly, decreased plasma levels of miR-222, miR-151-5p, and let-7e after thyroidectomy in papillary thyroid cancer patients indicate their secretion in systemic circulation by tumors (18). In this context, biomarker potential of circulating miRNAs was shown for multiple diseases, including reproductive diseases $(19,20)$.

In contrast to humans, very few research studies showed the biomarker potential of circulating miRNAs in farm animals. miRNAs have been shown to be involved in reproductive physiology in animals, including regulation of follicular and luteal development (21), pregnancy (22), and follicle-to-luteal transition (23). Moreover, it has been reported that the miRNA profile of the reproductive tissues $(11,23,24)$ and plasma of bovine varies in different phases of an estrus cycle (9), which could be due to cyclic variation in ovarian hormones, mainly estrogen and progesterone. As the ovary is highly vascularized organ (9), it is reasonable to hypothesize that miRNAs from ovarian tissue may be excreted differentially and/or specifically in circulation, depending on the phase of an estrus cycle, suggesting that circulating miRNAs may mirror ovarian miRNA profile. In addition, some of these systemic miRNAs may get filtered out via the kidney and ultimately appear in urine, and hence, they can be used as estrus biomarkers. Thus, circulating miRNAs in bovine urine may convey the specific phase of an estrous cycle.

Although miRNA profile in bovine plasma (9) and ovarian tissues $(23,25-27)$ gets altered during an estrous cycle, no study has been conducted until now to identify the altered levels of urinary miRNAs during bovine estrous cycle. Therefore, the present study was planned to explore the altered levels of urinary miRNA during estrous cycle considering the buffalo as a model. In this study, we used quantitative reverse transcriptasepolymerase chain reaction (qRT-PCR) to detect the levels of 10 hormone-responsive miRNAs, which were selected on the basis of their implication in ovarian physiology as per the available scientific literature, i.e., miR-125b (28), miR-99a (29), miR145 (30), miR-21 (31), miR-191 (30), miR-210 (32), let-7f (30), miR-16 (33), miR-378 (30), and miR-126 (34) in buffalo urine and compared their levels across different phases of an estrous cycle. In addition, validation of urinary miR-99a-5p levels was performed in a separate group of 25 buffaloes. At last, in silico analysis was done to identify putative targets of miR-99a-5p using miRwalk 2.0 followed by their association analysis with different cellular signaling pathways using Panther, an online tool.

\section{MATERIALS AND METHODS}

\section{Experimental Animals}

For the discovery phase of the study, three buffalo heifers were managed as per the standard conditions at the Livestock Research Centre, ICAR-National Dairy Research Institute (NDRI), Karnal. In addition, 25 animals, which were presented for AI at the AI center, ICAR-NDRI, were considered for the validation study. The study was approved by the NDRI Institutional Animal Ethics Committee (approval no. 42-IAEC-18-2).

\section{Sample Collection and Processing}

Urine and saliva samples were collected in the morning time and in the evening on the day of $\mathrm{E}$ (day 0), proestrus (PE) (day -2), and diestrus (DE) (day 10). In case animals did not urinate for $20 \mathrm{~min}$, the animals were stimulated for urination by pouring water on the rump region or by tying the animal or by offering the water to the animal. In brief, midstream urine was collected from healthy buffalo heifers $(n=3$ for discovery group and $n=25$ for validation group) in $50 \mathrm{~mL}$ centrifuge tubes, transported to the laboratory on ice, and centrifuged at 3,000 $\mathrm{g}$ for $5 \mathrm{~min}$ at $4^{\circ} \mathrm{C}$. The supernatant or cell-free urine (CFU) was transferred to another microcentrifuge tube and then either used immediately or stored in $-20^{\circ} \mathrm{C}$ until further use. In case of the discovery group, urine was collected from different phases of three consecutive estrous cycles of each animal, but the samples that belonged to the middle estrous cycle were selected for further analysis. In validation group, urine was collected from buffaloes on day 0 before insemination and again on day 10. Estrus and DE paired samples from animals that showed typical salivary fern pattern of estrus on day 0 and were found to be non-pregnant during 60 days after AI were included in this study.

\section{Detection of an Estrous Cycle Phase}

Buffaloes were observed for estrus symptoms twice a day in the morning and the evening for the period of 6 months. E phase was determined by observing various signs such as visual observation (vaginal discharge, hyper salivation, and abnormal posture), gynecoclinical examination (cervical relaxation and 
uterine tonicity), and biochemical confirmation (cervical mucus crystallization or salivary fern pattern) and rated as either mild, moderate, or intense as mentioned in Table 2. Serum $(n=$ 3) was used to estimate the estradiol levels using enzymelinked immunosorbent assay-based Estradiol Estimation Assay kit (ADI-900-174 by ENZO) as per the manufacturer's protocol.

\section{Saliva Collection and Fern Pattern Analysis}

Unstimulated saliva was collected from the lower lip of buffaloes before feeding time in the morning on every alternative day of the estrous cycle, brought to the laboratory on ice and centrifuged at $3,000 \mathrm{~g}$ for $5 \mathrm{~min}$ at $4^{\circ} \mathrm{C}$. A $10 \mu \mathrm{L}$ of cell-free saliva was used for smear preparation on a clean glass slide for observing the salivary fern patterns under an inverted microscope (Nikon Eclipse Ti$\mathrm{S}$, Japan) to determine the phase of an estrous cycle as per our previous study (2) (Figure 1).

\section{Total RNA Isolation}

RNA was extracted from CFU using TRIzol LS (Life Technologies, USA) as per the manufacturer's protocol. In brief, $250 \mu \mathrm{L}$ of CFU was mixed with $750 \mu \mathrm{L}$ of TRIzol LS and vortexed briefly, and the mixture was kept at room temperature for $5 \mathrm{~min}$. Then, $200 \mu \mathrm{L}$ of chloroform (Sigma Chemicals Co., catalog no. C2432) was added to the mixture, vortexed for $15 \mathrm{~s}$, and incubated for $15 \mathrm{~min}$ at room temperature followed by a centrifugation at 12,000 relative centrifugal force ( $\mathrm{rcf}$ ) for $15 \mathrm{~min}$. Aqueous supernatant was transferred to a fresh Eppendorf tube, $500 \mu \mathrm{L}$ of isopropanol was added to it, and the mixture was incubated at $-20^{\circ} \mathrm{C}$ for $30 \mathrm{~min}$. Later, it was centrifuged at $12,000 \mathrm{rcf}$ for $10 \mathrm{~min}$. The supernatant was discarded, and the pellet was washed with $70 \%$ ethanol. Finally, the RNA pellet was dissolved in $20 \mu \mathrm{L}$ of nuclease-free water and then either used immediately or stored at $-20^{\circ} \mathrm{C}$ until further use. RNA purity and concentrations were determined by using a Nanodrop spectrophotometer.

\section{cDNA Synthesis}

Total RNA was used to prepare cDNA as per the miScript II RT kit (catalog no. 218161, Germany) according to the manufacturer's protocol. In brief, $10 \mu \mathrm{L}$ of cDNA reaction mixture containing $500 \mathrm{ng}$ of the total RNA, $2 \mu \mathrm{L}$ of $5 \mathrm{X}$
miScript Hispec buffer, $1 \mu \mathrm{L}$ of RT mix, $1 \mu \mathrm{L}$ of $10 \times$ miScript nucleics mix provided by Qiagen miscript II kit (catalog no. 218161, Germany), and $1 \mu \mathrm{L}$ of an exogenous spiked-in miRNA control, syn-cel-miR-39-3p (1 $\mu \mathrm{L}$; miRNeasy Serum/Plasma Spike-In Control, catalog no. 219610, Qiagen Co., Germany) was incubated at $37^{\circ} \mathrm{C}$ for $60 \mathrm{~min}$ and $95^{\circ} \mathrm{C}$ for $5 \mathrm{~min}$. The prepared cDNA was kept at $-20^{\circ} \mathrm{C}$ until further use.

\section{Real-Time PCR}

Urinary levels of miRNAs were determined by qRT-PCR with some modifications using either Light Cycler 480 II (Roche, CA, USA) or Applied Biosystems Fast 7500 Real-Time PCR system (Roche, CA, USA). The primers used for miRNA amplification were designed on the basis of mature miRNA sequences of bovine using miRBase 21 and procured from a commercial firm (Table 1) (35). In brief, $12 \mu \mathrm{L}$ PCR reaction mixture consisting of $5 \mu \mathrm{L}$ of cDNA (1:20 diluted), $5 \mu \mathrm{L}$ of $2 \times$ Quantilect SYBR Green PCR Master Mix, $1 \mu \mathrm{L}$ of $5 \mu \mathrm{M}$ miRNA-specific primer, and $1 \mu \mathrm{L}$ of $10 \times$ miScript Universal Primer (catalog no. 218073, Germany) was incubated at $95^{\circ} \mathrm{C}$ for $15 \mathrm{~min}$, followed by 40 cycles of $94^{\circ} \mathrm{C}$ for $15 \mathrm{~s}, 55^{\circ} \mathrm{C}$ for $30 \mathrm{~s}$, and $70^{\circ} \mathrm{C}$ for $30 \mathrm{~s}$. The melt curve analysis was performed at the temperature ranging from 70 to $95^{\circ} \mathrm{C}$. Each sample was run in duplicate. qRT-PCR data analysis was done using $2^{-\Delta \Delta C t}$ method (36) by using cel-miR-39 as an exogeneous spiked-in miRNA for normalization (13).

TABLE 1 | miRNAs primers used in the study.

\begin{tabular}{lll}
\hline Gene name & Primer name: sequence $\mathbf{( 5}^{\prime}$ to $\mathbf{3}^{\prime} \mathbf{)}$ & Accession $\mathbf{n o .}$ \\
\hline $\begin{array}{ll}\text { bta-miR-99a-5p } \\
\text { bta-mir-145 }\end{array}$ & AACCCGTAGATCCGTTCTTGT & MIMAT0003537 \\
bta-mir-125b & TCCCAGTTTCCCAGGAATCCC & MIMAT0003542 \\
bta-mir-126-3p & CGTACCGTGAGTAATAATGCG & MIMAT0003539 \\
bta-miR-21-5p & GCTTATCAGACTGATGTTGAC & MIMAT0003528 \\
bta-let-7f & TGAGGTAGTAGATTGTATAGTT & MIMAT0003519 \\
bta-mir-210 & ACTGTGCGTGTGACAGC & MIMAT0003824 \\
bta-mir-378 & ACTTGGAGTCAGAAGGC & MIMAT0009305 \\
bta-miR-191 & CAACGGAATCCCAAAAG & MIMAT0003819 \\
bta-mir-16b & TAGCAGCACGTAAATATTGG & MIMAT0003525
\end{tabular}
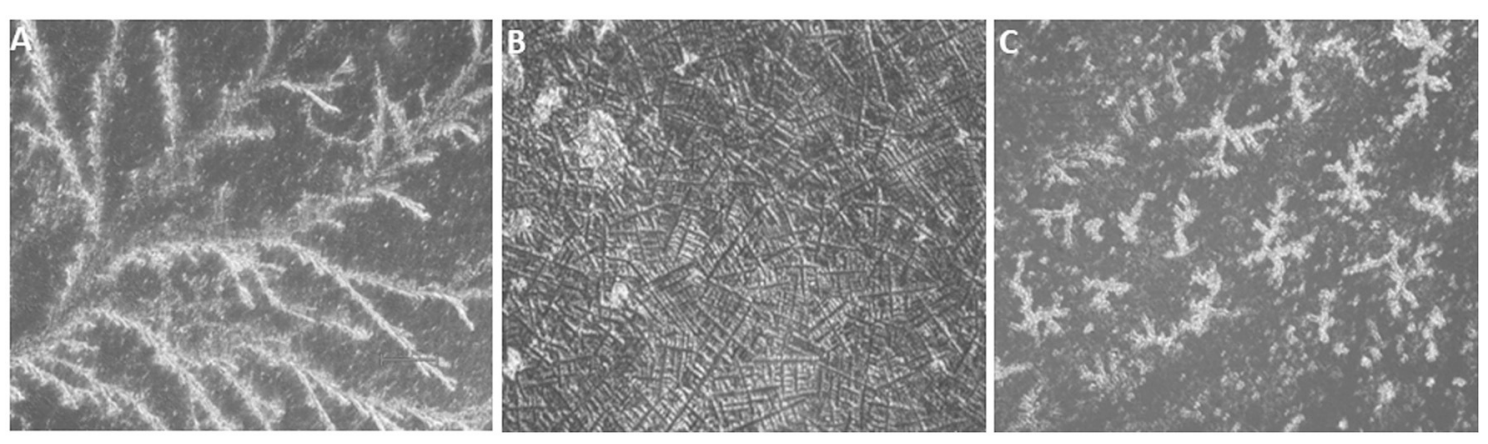

FIGURE 1 | Typical salivary crystallization of buffalo (air dried, ×200). (A) Salivary crystallization during the proestrus phase. (B) Typical fern patterns of saliva during the estrus phase. (C) Discontinuous and improper crystallization during the diestrus phase. 
TABLE 2 | Intensities of overt estrous signs in different buffaloes.

\begin{tabular}{lccc}
\hline Animal no. & A1 & A2 & A3 \\
\hline Swollen vulva & + & ++ & ++ \\
Vaginal discharge & ++ & ++ & +++ \\
Salivary fern & ++ & ++ & +++ \\
Tonicity of uterus & ++ & ++ & ++ \\
\hline
\end{tabular}

Estrus intensities were determined by visual behaviors and biochemical and gynecoclinical parameter as mild $(+)$, moderate $(++)$, and intense $(+++)$.

TABLE 3 | Estradiol levels during different phases of an estrous cycle in buffaloes.

\begin{tabular}{lccccc}
\hline Animal no. & A1 & A2 & A3 & Average & SE \\
\hline PE & 26.9 & 27.08 & 34.76 & 29.58 & 2.591 \\
E & 21.35 & 16.87 & 25.96 & 21.39 & 2.624 \\
DE & 14.39 & 16.01 & 18.43 & 16.28 & 1.174 \\
\hline
\end{tabular}

\section{Target Gene Prediction and Pathway Analysis for miR-99a-5p}

MiRWalk, an online tool, was used to predict putative mRNA targets that might be regulated by miR-99a-5p under a threshold of 3 (37). The resultant predicted targets for miR-99a-5p were run through Protein Analysis Through Evolutionary Relationship (PANTHER) classification system and analysis tools (38) in order to identify their associated biological processes considering the bovine as a genome background.

\section{Statistical Analysis}

Statistical analyses were performed either by one-way analysis of variance (ANOVA) followed by post-hoc Tukey test or paired $t$-test using GraphPad Prism software 5.1. (GraphPad Software, Inc., San Diego, CA, USA). Results are shown as the mean \pm SEM. Receiver operating characteristic (ROC) curve analysis was performed using $\Delta \mathrm{Ct}$ values of the miR-99a-5p in the $\mathrm{E}$ and DE phase samples.

\section{RESULTS}

\section{Intensity of Estrus Signs}

Estrus was determined by various physical indicators such as tonicity of uterus, swelling of vulva, vaginal discharge, and typical salivary fern patterns. Physical indicators were categorized as mild, moderate, and intense as shown in Table 2. Among three buffaloes at the E phase, moderate or intense swelling of vulva was recorded in $66.67 \%$ of cases together. Similarly, estrus intensity indicators such as vaginal discharge and salivary ferns were observed to be either moderate or intense in all animals (100\%). Tonicity of uterus was found to be moderate in $100 \%$ of the animals. Serum estradiol levels were further determined at E phase in buffaloes (Table 3), which were found to be significantly higher at the PE $(29.58 \pm 2.591 \mathrm{pg} / \mathrm{mL})$ and $\mathrm{E}$ phases $(21.39 \pm$ $2.624 \mathrm{pg} / \mathrm{mL} ; P<0.05)$ as compared to the DE phase $(16.28 \pm$ $1.174 \mathrm{pg} / \mathrm{mL})$.

\section{miRNA Urinary Levels}

We selected 10 hormone-responsive miRNA candidates identified on the basis of literature (Table 2) for qRT-PCR analysis. Unfortunately, the urinary levels of let-7f, mir-126-3p, miR-378, and mir-16b were too low to be detected by qRT-PCR. Of the remaining six miRNAs, mir-145 (1.5-fold E/PE; 0.7-fold $\mathrm{DE} / \mathrm{PE}$ ), mir-21(1.5-fold E/PE; 2-fold DE/PE), miR-210 (1.2fold E/PE; 0.7-fold DE/PE), mir-191 (1.3-fold E/PE; 0.8-fold $\mathrm{DE} / \mathrm{PE})$, and miR-125b (0.5-fold E/PE; 0.7-fold DE/PE) did not significantly change during the $\mathrm{E}$ cycle (Figure 2). In contrast, miR-99a-5p levels increased at the DE phase (1.9-fold) and decreased at the E phase $(0.5$-fold $P<0.05)$ compared to the $\mathrm{PE}$ phase as shown in Figure 2.

\section{Validation of mir-99a-5p Urinary Levels and ROC Curve Analysis}

mir-99a-5p levels were analyzed in another group of 25 buffaloes on the day of the $\mathrm{E}$ and DE phases during an estrous cycle. mir99a-5p levels were observed to be significantly increased (3.92fold; $P<0.0001$ ) in the DE phase as compared to the $\mathrm{E}$ phase as shown in Figure 3. Finally, we determined discriminatory performance of miR-99a-5p using ROC curve analysis as shown in Figure 4. E and DE datasets were used to make ROC curve. ROC curve analysis showed that at the 7.962 cutoff value, the sensitivity and specificity of miR-99a-5p in differentiating the E from DE phase were 56 and 60\%, respectively; the AUC was 0.6464 (95\% confidence interval, $0.4921-0.8007 ; P=0.076$ ) as shown in Figure 4. ROC curve results suggest the potential of miR-99a-5p to distinguish the $\mathrm{E}$ and DE phases.

\section{Target Gene Prediction and Pathway Analysis of miR-99a-5p}

miRwalk, an online tool, predicted 7,347 target genes for miR-99a-5p (Supplementary Table 1). miR-99a-5p target sites were identified in the $5^{\prime}$ UTR, CDs, and $3^{\prime}$ UTR regions of putative targets. Among 7,347 predicted target genes, 3,086 putative target genes having NM_Accession prefixes (Supplementary Table 2) were shown to be associated with various cellular pathways (Supplementary Table 3) and biological processes (Supplementary Table 4) by the PANTHER software. Among the top 15 cellular pathways predicted to be regulated by miR-99a-5p, androgen/estrogen/progesterone biosynthesis and apoptosis signaling pathways have been previously implicated in ovarian physiology (Figure 5). Similarly, biological process annotations by PANTHER analysis showed that miR-99a-5p may regulate reproduction and reproductive process (Figure 6).

\section{DISCUSSION}

Currently, an accurate estrus detection biomarker is the need of the hour for effective reproductive management of buffaloes. Recently, a number of studies reported biomarker potential of circulating miRNAs in biofluids owing to their high stability (39) and good clinical performance (40). Among the various miRNAs evaluated in the present study, we found low levels of miR-99a-5p and bta-mir-125b on the estrus day as compared 


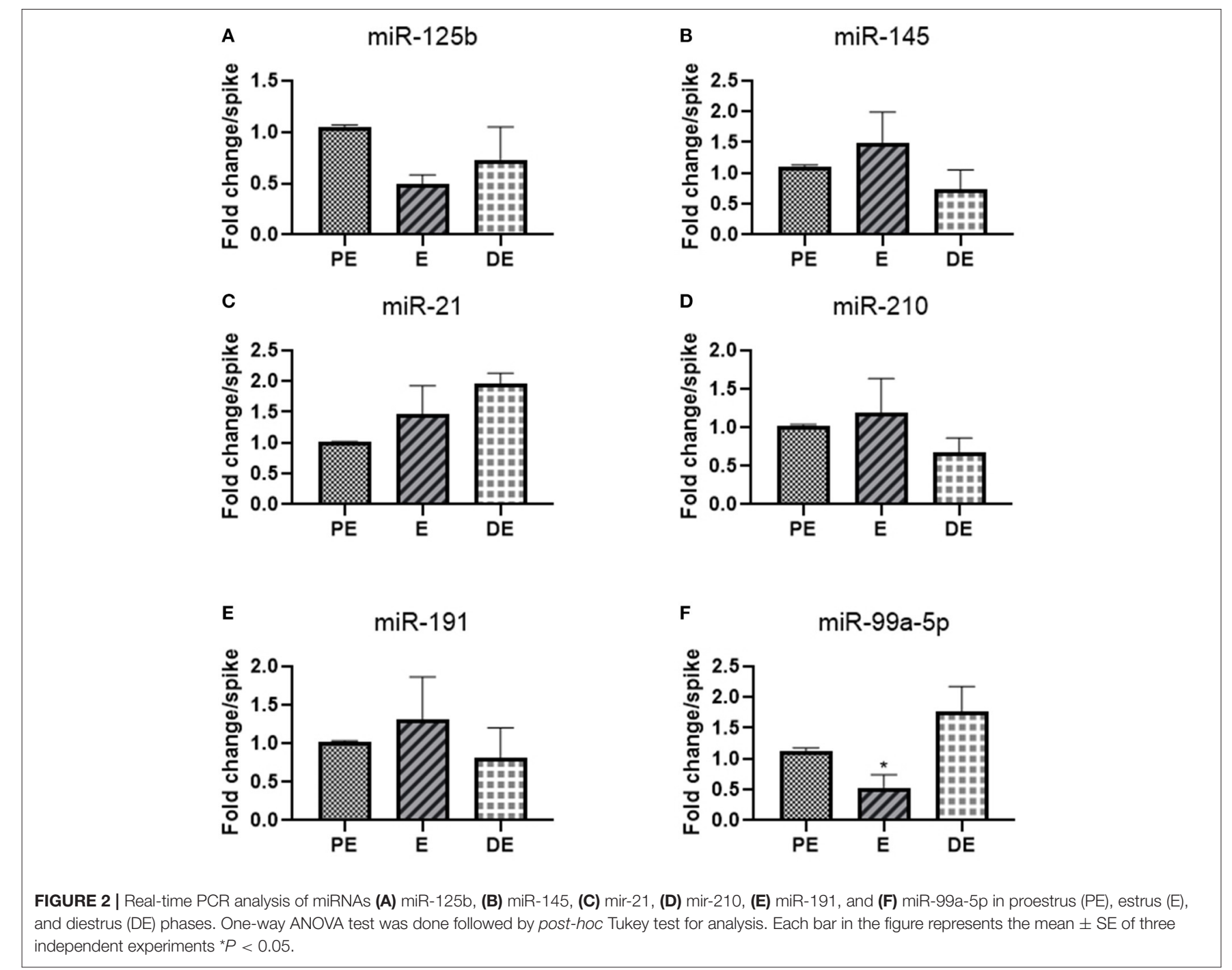

to the PE and DE phases. In contrast, earlier studies showed a higher expression of miR-99a-5p and bta-mir-125b in ovaries during follicular phase and their higher plasma levels during the E phase in cows (9). Another study reported an upregulation of miR-125b expression by androgens, thereby suppressing the follicular atresia via targeting a proapoptotic gene (28). Moreover, decreased expression of mir-125b was found in granulosa cells of primordial follicles as compared to primary, secondary, and antral follicles in mouse (41).

In addition, we also found high levels of mir-145, mir210, and mir-191 on the estrus day as compared to the PE phase. In concordance with our data, higher expression of mir$145(23,25)$ and mir-210 (32) were shown in ovaries during follicular phase, and higher plasma levels of mir-145 were reported during the E phase of cows (9). Furthermore, our study showed a gradual increase of miR-21 levels from the $\mathrm{PE}$ to the $\mathrm{E}$ phase, followed by its highest levels at the DE phase, which is not in agreement with earlier studies as miR21 expression in the granulosa cells was found to be lowest on day 14 and at higher levels on day 3 of an estrous cycle (42). Study done by Donadeu et al. (43) reported higher expression of miR-21-5p/-3p in atretic than healthy follicles of cattle. In this context, studies reported the regulation of follicular atresia by miR-21-3p through targeting FGF2 (44) and VEGFA (45) that ultimately inhibits bovine granulosa cell autophagy by repressing AKT/mTOR signaling and PI3K/AKT signaling, respectively. Another study reported the higher expression of bta-miR-21$5 \mathrm{p}$ during early CL (1-7 days) as compared to middle CL and late CL (46), suggesting its role in the corpus luteum formation, functionalization, and corpus luteum regression (23). Luteinizing hormone was reported to upregulate antiapoptotic miR-21 in murine granulosa cells, suggesting its role in granulosa cell survival and differentiation (47). Moreover, elevated levels of miR-21, miR-145, and miR-378 in follicular fluid aspirated from dominant follicles during seasonal anovulation as compared to the seasonal ovulation period in mares further suggest their role in follicle maturation (48). Literature survey also showed the higher levels of miR-125b $(23,27)$ and miR-145 $(23,25)$ and low 


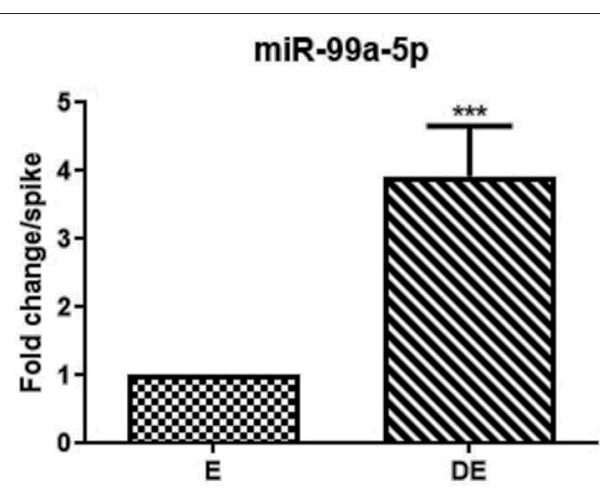

FIGURE 3 | Real-time PCR analysis of mir-99a-5p in the validation population of the animals in estrus (E) and diestrus (DE). The paired $t$-test was used for analysis. Each bar represents the mean \pm SEM of 25 independent experiments. ${ }^{* \star \star} P<0.001$.

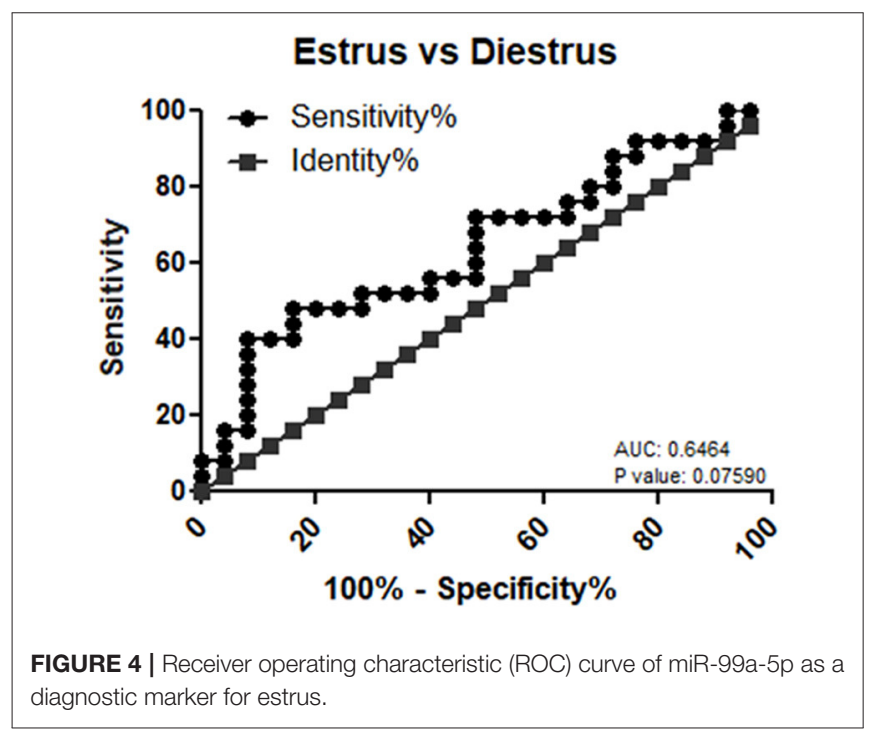

levels of miR-21 $(23,26)$ in follicular tissues as compared to luteal tissues in the ovaries, indicating their role in follicle-to-luteal transition. At last, let-7f, miR-16b, miR-126-3p, and mir-378 were not detected in buffalo urine, suggesting their levels might be below the detection limit of qRT-PCR or completely absent. In this context, Weber et al. (49) reported that urine generally has the lowest amount of miRNAs among 12 different biological fluids.

Hormone plays an important role in estrous cycle regulation. In this context, multiple studies showed a reciprocal relationship between miRNAs expression/levels and hormones. One of the studies suggested partial regulation of circulating miR-125b, miR99a, miR-145, and miR-378 by hormones in hyperstimulated heifers (50). Hu et al. (51) reported downregulation of miR$125 \mathrm{~b}$ expression in response to $17 \alpha-\mathrm{E} 2$. Reciprocally, miRNAs also regulate ovarian hormone biosynthesis and release. Sirotkin et al. (52) demonstrated the role of miRNAs in the regulation of estradiol (miR-125b and miR-126), testosterone (miR-16,
miR-145, miR-125b, miR-21, and miR-126), and progesterone (miR-16, miR-145, miR-125b, and miR-126) release, respectively, in granulosa cells. Zhang et al. (53) reported that decreased expression of miR-125b-5p stimulates testosterone secretion and decreases the estradiol release in mouse preantral follicles via regulation of PAK3/ERK1/2 signaling. Hence, it can be concluded that miRNAs and hormonal interplay might be involved in estrous cycle regulation. Moreover, multiple investigations showed the extracellular presence of the miRNAs evaluated in the present study. Naji et al. (54) reported that higher levels of miR-145 in follicular fluid can be used as a predictive biomarker for polycystic ovary syndrome. Singh et al. (55) reported the lower levels of salivary mir-16b in the presence of dominant ovarian follicle. Li et al. (34) reported higher serum levels of miR-126-3p during ovulation and midluteal phase in comparison to early follicular phase. A study conducted with cow plasma demonstrated the significantly increased levels of miR-99a-5p, let-7f, miR-145, and miR-125b in estrus as compared to the other phases during estrous cycle of cows (9). Taken together, the miRNA presence in biofluids further suggests their potential use as a biomarker.

Among the studied miRNAs, miR-99a-5p can be used as an estrus biomarker. Tripurani et al. (56) reported that the higher expression of miR-99a in ovarian tissue suggested its role in basic reproductive activities. Geng et al. (57) reported decreased proliferation and increased apoptosis of granulosa cells by miR-99a via targeting insulin like growth factor $1 \mathrm{R}$, suggesting its expression might be low during folliculogenesis, which could partly explain its low urinary levels during estrus in buffaloes. Literature survey also indicated the role of miR99a in cell cycle (58) and glucose metabolism (59). Noferesti et al. (50) reported hormonal regulation of circulating miR99a, which further explains its dynamic urinary levels during an estrous cycle due to hormonal cyclicity. The functions of miR-99a-5p have not been reported completely as yet in relation to fertility and physiological functions in the ovary; we herein speculate its possible role by predicting the association of miR99a-5p targets with different cellular pathways, including those pathways associated with biosynthesis of androgen, estrogen, and progesterone hormones and apoptosis, which ultimately suggests its role in the reproductive process. Hence, in silico analysis gives clues regarding the implication of miR-99a-5p in ovarian physiology. Moreover, ROC curve analysis gave a solid support that miR-99a-5p could clearly differentiate between the $\mathrm{E}$ and $\mathrm{DE}$ phase. At last, its dynamic presence in plasma and urine during the estrous cycle as shown previously further hints toward its definite potential as an estrus biomarker in the near future. Thus, these data explained why miR-99a could serve as a promising biomarker for estrus detection in buffaloes.

In general context, lack of agreement between the present and numerous previous studies might be due to difference in preanalytical and analytical variables used, including sample source, RNA isolation protocols, RNA quality and quantity, cDNA synthesis kits, miRNA quantification techniques, and normalization gene $(60,61)$. For example, miRNA profile varies according to different body fluids. For example, an miRNA 


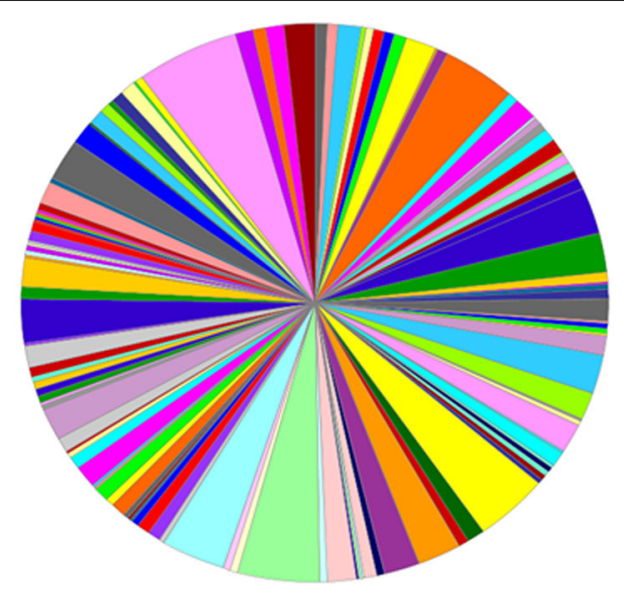

PANTHER PATHWAY
5- -Hydroxytryptamine degredation (P04372)
$5 \mathrm{HT} 1$ type receptor mediated signaling pathway (P04373)
$5 \mathrm{HT} 2$ type receptor mediated signaling pathway (P04374)
$5 \mathrm{HT} 3$ type receptor mediated signaling pathway (P04375)
5HT4 type receptor mediated signaling pathway (P04376)
Adrenaline and noradrenaline biosynthesis (P00001)
Alpha adrenergic receptor signaling pathway (P00002)
Alzheimer disease-amyloid secretase pathway (P00003)
Alzheimer disease-presenilin pathway (P00004)
Aminobutyrate degradation (P02726)
Androgen/estrogene/progesterone biosynthesis (P02727)
Angiogenesis (P00005)
Angiotensin II-stimulated signaling through G proteins and beta-arrestin (P05911)
Apoptosis signaling pathway (P00006)
Asparagine and aspartate biosynthesis (P02730)

FIGURE 5 | Association of bta-miR-99a-5p target genes with different pathways as shown in the pie chart by PANTHER, an online tool.

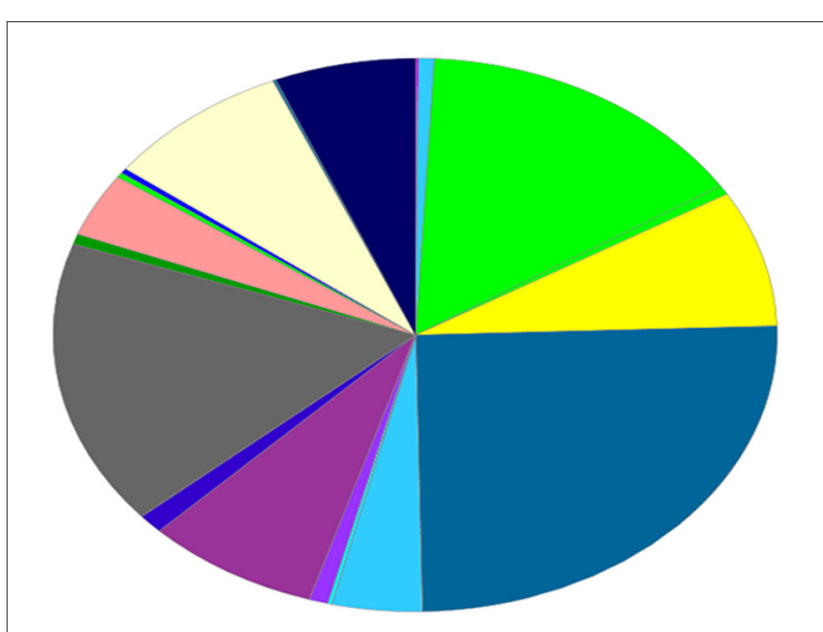

\begin{tabular}{l} 
PANTHER GO-Slim Bioloqical Process \\
\hline behavior (GO:0007610) \\
biological adhesion (GO:0022610) \\
biological regulation (GO:0065007) \\
cell population proliferation (GO:0008283) \\
cellular component organization or biogenesis (GO:0071840) \\
cellular process (GO:0009987) \\
developmental process (GO:0032502) \\
growth (GO:0040007) \\
immune system process (G0:0002376) \\
localization (GO:0051179) \\
locomotion (GO:0040011) \\
metabolic process (GO:0008152) \\
multi-organism process (G0:0051704) \\
multicellular organismal process (GO:0032501) \\
reproduction (G0:0000003) \\
reproductive process (GO:0022414) \\
response to stimulus (GO:0050896) \\
rhythmic process (GO:0048511) \\
signaling (GO:0023052)
\end{tabular}

FIGURE 6 | Association of bta-miR-99a-5p target genes with different biological processes as shown in the pie chart by PANTHER, an online tool.

profile in urine and plasma samples from the same animal may vary, as urine being the ultrafiltrate of plasma. In addition, we stored CFU in $-20^{\circ} \mathrm{C}$, which may affect RNA yield, as urinary Extracellular Vesicles (EVs) or naked miRNAs may get entrapped inside Tamm-Horsfall protein complex present in the urine under low temperature conditions and subsequently get lost during RNA isolation (62). Currently, there is no consensus regarding ideal reference gene that should be used for qRT-PCR data normalization in case of biofluids. In the present study, exogenous cel-mir-39 was used for qRT-PCR data normalization, which may reduce variability caused by differences in reverse transcription efficiency across samples, but it is insufficient to remove technological variability across samples caused by various factors including collection, handling, and storage of samples. At last, cell-free miRNA profile and their levels in urine are dynamic in nature and depend on various factors, including intervariability and intravariability among animals, time point of urine collection, and seasonal variation. Hence, there is always a chance of getting variable miRNA profile from urine taken at the same phase of an estrous cycle.

Our study has a number of limitations. First, our study is not comprehensive in terms of cell-free miRNAs profiling present in buffalo urine, although it showed the biomarker potential of urinary miR-99a-5p for estrus detection. Second, we used a small number of samples during the discovery and validation phase; hence, our finding needs to be verified in a large sample size. Third, our results must be verified in different breeds of buffaloes. Fourth, urinary levels of miR-99a-5p were found to be differentially altered at different phases of estrous cycle in buffalo, but the origin of urinary miR-99a-5p needs to be elucidated in order to draw a significant conclusion regarding its biomarker potential, as it is already reported to be expressed in numerous other tissues (56) apart from the ovary. Hence, the relative contribution of these organs to urinary miR-99a-5p is not known. Similarly, cell-specific expression of miR-99a-5p in ovarian tissue must be determined in order to better estimate its potential 
as an estrus biomarker. Fifth, it needs to be deciphered how miR-99a-5p enters the circulation, especially the urine.

In the future, successful clinical use of urinary cellfree miRNAs requires an establishment of guidelines and protocols for urine sampling, processing, storage, and transport; miRNA isolation, normalization, and quantification methods; and miRNA profiling using high-throughput detection techniques. Meanwhile, urinary miRNA diversity and their levels in different phases of an estrous cycle need to be determined for the development of estrus detection biomarker in buffaloes. At last, the biomarker potential of urinary cell-free mir-99a-5p needs to be deciphered in combination with other miRNA panels to increase its sensitivity and specificity for heat detection in the buffalo.

In summary, our study yielded (i) a first insight of hormone-responsive miRNAs levels in buffalo urine using real-time PCR, (ii) a potential of urinary cell-free miR-99a$5 \mathrm{p}$ as estrus biomarker in bovine, and (iii) a clue that ovarian-derived miRNAs may get filtered out in buffalo urine. Most importantly, ROC curve analysis showed that urinary levels of miR-99a-5p in buffalo can moderately distinguish the $\mathrm{E}$ from DE phases of an estrous cycle, further suggesting its biomarker potential in estrus detection that needs to be further explored in future either alone or in combination with other miRNAs to improve its sensitivity and specificity.

\section{CONCLUSION}

The present study reported significantly lower levels of urinary cell-free mir-99a-5p at the $\mathrm{E}$ phase as compared to the DE phase in buffalo. This may be helpful for the

\section{REFERENCES}

1. Mondal S, Prakash BS, Palta P. Endocrine aspects of oestrous cycle in buffaloes (Bubalus bubalis): an overview. Asian-Australasian J Anim Sci. (2007) 20:12431. doi: 10.5713/ajas.2007.124

2. Ravinder R, Kaipa O, Baddela VS, Singhal Sinha E, Singh P, Nayan V, et al. Saliva ferning, an unorthodox estrus detection method in water buffaloes (Bubalus bubalis). Theriogenology. (2016) 86:1147-55. doi: 10.1016/j.theriogenology.2016.04.004

3. Suthar VS, Dhami AJ. Estrus detection methods in buffalo. Vet World. (2010) 3:94-6.

4. De Rensis F, López-Gatius F. Protocols for synchronizing estrus and ovulation in buffalo (Bubalus bubalis): a review. Theriogenology. (2007) 67:20916. doi: 10.1016/j.theriogenology.2006.09.039

5. Verma KK, Prasad S, Kumaresan A, Mohanty TK, Layek SS, Patbandha TK, et al. Characterization of physico-chemical properties of cervical mucus in relation to parity and conception rate in Murrah buffaloes. Vet World. (2014) 7:467-71. doi: 10.14202/vetworld.2014.4 67-471

6. Selvam RM, Onteru SK, Nayan V, Sivakumar M, Singh D, Archunan G. Exploration of Luteinizing hormone in murrah buffalo (Bubalus bubalis) urine: extended surge window opens door for estrus prediction. Gen Comp Endocrinol. (2017) 251:121-6. doi: 10.1016/j.ygcen.2016. 12.002 development of mir-99a-5p-based estrus detection kit in the near future.

\section{DATA AVAILABILITY STATEMENT}

The datasets presented in this study can be found in online repositories. The names of the repository/repositories and accession number(s) can be found in the article/Supplementary Material.

\section{ETHICS STATEMENT}

The animal study was reviewed and approved by IAEC, NDRI, Karnal.

\section{AUTHOR CONTRIBUTIONS}

$\mathrm{AH}$ and PR: performed the research, methodology, and data analysis. RC: writing-original draft. SO: conceptualization and supervision. DS: conceptualization, supervision, and experimental design. All authors contributed to the article and approved the submitted version.

\section{FUNDING}

This work was supported by the Bill \& Melinda Gates Foundation [grant number OPP1154401] .

\section{SUPPLEMENTARY MATERIAL}

The Supplementary Material for this article can be found online at: https://www.frontiersin.org/articles/10.3389/fvets. 2021.643910/full\#supplementary-material

7. Roelofs J, López-Gatius F, Hunter RHF, van Eerdenburg FJCM, Hanzen C. When is a cow in estrus? Clinical and practical aspects. Theriogenology. (2010) 74:327-44. doi: 10.1016/j.theriogenology.2010.02.016

8. Selvam RM, Archunan G. A combinatorial model for effective estrus detection in Murrah buffalo. Vet World. (2017) 10:20913. doi: 10.14202/vetworld.2017.209-213

9. Ioannidis J, Donadeu FX. Circulating microRNA profiles during the bovine oestrous cycle. PLoS ONE. (2016) 11: e0158160. doi: 10.1371/journal.pone.0158160

10. Rao TKS, Kumar N, Kumar P, Chaurasia S, Patel NB. Heat detection techniques in cattle and buffalo. Vet World. (2013) 6:363-9. doi: 10.5455/vetworld.2013.363-369

11. Sontakke SD, Mohammed BT, McNeilly AS, Donadeu FX. Characterization of microRNAs differentially expressed during bovine follicle development. Reproduction. (2014) 148:271-83. doi: 10.1530/REP-14-0140

12. Jerome A, Thirumaran SMK, Kala SN. Identification of microRNAs in corpus luteum of pregnancy in buffalo (Bubalus bubalis) by deep sequencing. Iran J Vet Res. (2017) 18:287-90. doi: 10.14202/vetworld.2017.1129-1134

13. Mitchell PS, Parkin RK, Kroh EM, Fritz BR, Wyman SK, PogosovaAgadjanyan EL, et al. Circulating microRNAs as stable blood-based markers for cancer detection. Proc Natl Acad Sci USA. (2008) 105:105138. doi: 10.1073/pnas.0804549105

14. Arroyo JD, Chevillet JR, Kroh EM, Ruf IK, Pritchard CC, Gibson DF, et al. Argonaute2 complexes carry a population of circulating microRNAs 
independent of vesicles in human plasma. Proc Natl Acad Sci USA. (2011) 108:5003-8. doi: 10.1073/pnas.1019055108

15. Vickers KC, Palmisano BT, Shoucri BM, Shamburek RD, Remaley AT. MicroRNAs are transported in plasma and delivered to recipient cells by highdensity lipoproteins. Nat Cell Biol. (2011) 13:423-33. doi: 10.1038/ncb2210

16. Williams Z, Ben-Dov IZ, Elias R, Mihailovic A, Brown M, Rosenwaks Z, et al. Comprehensive profiling of circulating microRNA via small RNA sequencing of cDNA libraries reveals biomarker potential and limitations. Proc Natl Acad Sci USA. (2013) 110:4255-60. doi: 10.1073/pnas. 1214046110

17. Petrozza V, Costantini M, Tito C, Giammusso LM, Sorrentino V, Cacciotti $\mathrm{J}$, et al. Emerging role of secreted miR-210-3p as potential biomarker for clear cell Renal Cell Carcinoma metastasis. Cancer Biomarkers. (2020) 27:1818. doi: $10.3233 / \mathrm{CBM}-190242$

18. Yu S, Liu Y, Wang J, Guo Z, Zhang Q, Yu F, et al. Circulating microRNA profiles as potential biomarkers for diagnosis of papillary thyroid carcinoma. J Clin Endocrinol Metab. (2012) 97:2084-92. doi: 10.1210/jc.2011-3059

19. Wu L, Zhou H, Lin H, Qi J, Zhu C, Gao Z, et al. Circulating microRNAs are elevated in plasma from severe preeclamptic pregnancies. Reproduction. (2012) 143:389-97. doi: 10.1530/REP-11-0304

20. Miura K, Higashijima A, Murakami Y, Tsukamoto O, Hasegawa Y, Abe S, et al. Circulating chromosome 19 miRNA cluster microRNAs in pregnant women with severe pre-eclampsia. J Obstet Gynaecol Res. (2015) 41:152632. doi: $10.1111 /$ jog. 12749

21. Donadeu FX, Schauer SN, Sontakke SD. Involvement of miRNAs in ovarian follicular and luteal development. J Endocrinol. (2012) 215:32334. doi: 10.1530/JOE-12-0252

22. Wessels JM, Edwards AK, Khalaj K, Kridli RT, Bidarimath M, Tayade C. The microRNAome of pregnancy: deciphering miRNA networks at the maternal-fetal interface. PLoS ONE. (2013) 8: e72264. doi: 10.1371/journal.pone.0072264

23. McBride D, Carré W, Sontakke SD, Hogg CO, Law A, Donadeu FX, et al. Identification of miRNAs associated with the follicular-luteal transition in the ruminant ovary. Reproduction. (2012) 144:221-33. doi: 10.1530/REP-12-0025

24. Salilew-Wondim D, Ahmad I, Gebremedhn S, Sahadevan S, Hossain $\mathrm{MM}$, Rings $\mathrm{F}$, et al. The expression pattern of microRNAs in granulosa cells of subordinate and dominant follicles during the early luteal phase of the bovine estrous cycle. PLOS ONE. (2014) 9: e106795. doi: 10.1371/journal.pone.0106795

25. Ling YH, Guo XF, Chen T, Ding JP, Ma YH, Chu MX, et al. Characterization and analysis of differentially expressed microRNAs in hircine ovaries during the follicular and luteal phases. Anim Reprod Sci. (2016) 166:4757. doi: 10.1016/j.anireprosci.2016.01.003

26. Zhu L, Chen T, Sui M, Han C, Fang F, Ma Y, et al. Comparative profiling of differentially expressed microRNAs between the follicular and luteal phases ovaries of goats. Springerplus. (2016) 5:1233. doi: 10.1186/s40064-016-2902-1

27. Zhang X, Zhang L, Shang J, Tao Q, Tian M, Ma Y, et al. Combined microRNAome and transcriptome analysis of follicular phase and luteal phase in porcine ovaries. Reprod Domest Anim. (2019) 54:1018-25. doi: 10.1111/rda.13457

28. Sen A, Prizant H, Light A, Biswas A, Hayes E, Lee HJ, et al. Androgens regulate ovarian follicular development by increasing follicle stimulating hormone receptor and microRNA-125b expression. Proc Natl Acad Sci USA. (2014) 111:3008-13. doi: 10.1073/pnas.1318978111

29. Xie J, Cao Y. Expression of TGF- $\beta 1$ and miR-99a in serum of patients with early spontaneous abortion and correlation with hormone levels during pregnancy. Exp Ther Med. (2019) 17:4593-7. doi: 10.3892/etm.2019.7477

30. Di Leva G, Piovan C, Gasparini P, Ngankeu A, Taccioli C, Briskin D, et al. Estrogen mediated-activation of miR191/425 cluster modulates tumorigenicity of breast cancer cells depending on estrogen receptor status. PLoS Genet. (2013) 9:e1003311. doi: 10.1371/annotation/92dfa670-d431-4d68-b70b-706df1f93e46

31. Wickramasinghe NS, Manavalan TT, Dougherty SM, Riggs KA, Li Y, Klinge CM. Estradiol downregulates miR-21 expression and increases miR-21 target gene expression in MCF-7 breast cancer cells. Nucleic Acids Res. (2009) 37:2584-95. doi: 10.1158/0008-5472.SABCS-3051

32. Shukla A, Dahiya S, Onteru SK, Singh D. Differentially expressed miRNA210 during follicular-luteal transition regulates pre-ovulatory granulosa cell function targeting HRas and EFNA3. J Cell Biochem. (2018) 119:793443. doi: $10.1002 / j \mathrm{cb} .26508$

33. Yu X, Zhang X, Dhakal IB, Beggs M, Kadlubar S, Luo D. Induction of cell proliferation and survival genes by estradiol-repressed microRNAs in breast cancer cells. BMC Cancer. (2012) 12:29. doi: 10.1186/1471-2407-12-29

34. Li P, Wei J, Li X, Cheng Y, Chen W, Cui Y, et al. 17 $\beta$-estradiol enhances vascular endothelial Ets-1/ MIR-126-3p expression: the possible mechanism for attenuation of atherosclerosis. J Clin Endocrinol Metab. (2017) 102:594603. doi: 10.1210/jc.2016-2974

35. Kozomara A, Griffiths-Jones S. MiRBase: annotating high confidence microRNAs using deep sequencing data. Nucleic Acids Res. (2014) 42:gkt1181. doi: 10.1093/nar/gkt1181

36. Livak KJ, Schmittgen TD. Analysis of relative gene expression data using realtime quantitative PCR and the 2- $\Delta \Delta$ CT method. Methods. (2001) 25:4028. doi: $10.1006 /$ meth.2001.1262

37. Dweep H, Gretz N, Sticht C. MiRWalk database for miRNAtarget interactions. Methods Mol Biol. (2014) 1182:289305. doi: 10.1007/978-1-4939-1062-5 25

38. Mi H, Huang X, Muruganujan A, Tang H, Mills C, Kang D, et al. PANTHER version 11: expanded annotation data from Gene Ontology and Reactome pathways, and data analysis tool enhancements. Nucleic Acids Res. (2017) 45:D183-9. doi: 10.1093/nar/gkw1138

39. Mall C, Rocke DM, Durbin-Johnson B, Weiss RH. Stability of miRNA in human urine supports its biomarker potential. Biomark Med. (2013) 7:10.2217/bmm.13.44. doi: 10.2217/bmm.13.44

40. Wang J, Chen J, Sen S. MicroRNA as biomarkers and diagnostics. J Cell Physiol. (2016) 231:25-30. doi: 10.1002/jcp.25056

41. Yao N. A network of miRNAs expressed in the ovary are regulated by FSH. Front Biosci. (2009) 3239:3447. doi: 10.2741/3447

42. Tian M, Zhang X, Ye P, Tao Q, Zhang L, Ding Y, et al. MicroRNA-21 and microRNA-214 play important role in reproduction regulation during porcine estrous. Anim Sci J. (2018) 89:1398-405. doi: 10.1111/asj.13087

43. Donadeu FX, Mohammed BT, Ioannidis J. A miRNA target network putatively involved in follicular atresia. Domest Anim Endocrinol. (2017) 58:76-83. doi: 10.1016/j.domaniend.2016.08.002

44. Ma L, Tang $\mathrm{X}$, Guo S, Liang $\mathrm{M}$, Zhang B, Jiang Z. miRNA-21-3p targeting of FGF2 suppresses autophagy of bovine ovarian granulosa cells through AKT/mTOR pathway. Theriogenology. (2020) 157:22637. doi: 10.1016/j.theriogenology.2020.06.021

45. Ma L, Zheng Y, Tang X, Gao H, Liu N, Gao Y, et al. MiR-21-3p inhibits autophagy of bovine granulosa cells by targeting VEGFA via PI3K/AKT signaling. Reproduction. (2019) 158:441-52. doi: 10.1530/REP-19-0285

46. Gecaj RM, Schanzenbach CI, Kirchner B, Pfaffl MW, Riedmaier I, TweedieCullen RY, et al. The dynamics of microRNA transcriptome in bovine corpus luteum during its formation, function, and regression. Front Genet. (2017) 8:213. doi: $10.3389 /$ fgene.2017.00213

47. Carletti MZ, Fiedler SD, Christenson LK. MicroRNA 21 blocks apoptosis in mouse periovulatory granulosa cells. Biol Reprod. (2010) 83:28695. doi: 10.1095/biolreprod.109.081448

48. Donadeu FX, Schauer SN. Differential miRNA expression between equine ovulatory and anovulatory follicles. Domest Anim Endocrinol. (2013) 45:1225. doi: 10.1016/j.domaniend.2013.06.006

49. Weber JA, Baxter DH, Zhang S, Huang DY, Huang KH, Lee MJ, et al. The microRNA spectrum in 12 body fluids. Clin Chem. (2010) 56:173341. doi: $10.1373 /$ clinchem. 2010.147405

50. Noferesti SS, Sohel MMH, Hoelker M, Salilew-Wondim D, Tholen E, Looft C, et al. Controlled ovarian hyperstimulation induced changes in the expression of circulatory miRNA in bovine follicular fluid and blood plasma. J Ovarian Res. (2015) 8:81. doi: 10.1186/s13048-015-0208-5

51. Hu Z, Shen WJ, Cortez Y, Tang X, Liu LF, Kraemer FB, et al. Hormonal regulation of microRNA expression in steroid producing cells of the ovary, testis and adrenal gland. PLoS ONE. (2013) 8:e78040. doi: 10.1371/journal.pone.0078040

52. Sirotkin AV, Ovcharenko D, Grossmann R, Lauková M, Mlynček M. Identification of microRNAs controlling human ovarian cell steroidogenesis via a genome-scale screen. J Cell Physiol. (2009) 219:415-20. doi: 10.1002/jcp.21689 
53. Zhang $\mathrm{X}$, Xiao $\mathrm{H}$, Zhang XEQ, Gong $\mathrm{X}$, Li T, Han $\mathrm{Y}$, et al. Decreased microRNA-125b-5p disrupts follicle steroidogenesis through targeting PAK3/ERK1/2 signalling in mouse preantral follicles. Metabolism. (2020) 107:154241. doi: 10.1016/j.metabol.2020.1 54241

54. Naji M, Nekoonam S, Aleyasin A, Arefian E, Mahdian R, Azizi E, et al. Expression of miR-15a, miR-145, and miR-182 in granulosa-lutein cells, follicular fluid, and serum of women with polycystic ovary syndrome (PCOS). Arch Gynecol Obstet. (2018) 297:221-31. doi: 10.1007/s00404-0174570-y

55. Singh P, Golla N, Singh P, Baddela VS, Chand S, Baithalu RK, et al. Salivary miR-16, miR-191 and miR-223: intuitive indicators of dominant ovarian follicles in buffaloes. Mol Genet Genomics. (2017) 292:93553. doi: $10.1007 / \mathrm{s} 00438-017-1323-3$

56. Tripurani SK, Xiao C, Salem M, Yao J. Cloning and analysis of fetal ovary microRNAs in cattle. Anim Reprod Sci. (2010) 120:1622. doi: 10.1016/j.anireprosci.2010.03.001

57. Geng Y, Sui C, Xun Y, Lai Q, Jin L. MiRNA-99a can regulate proliferation and apoptosis of human granulosa cells via targeting IGF1R in polycystic ovary syndrome. J Assist Reprod Genet. (2019) 36:21121. doi: $10.1007 / \mathrm{s} 10815-018-1335-\mathrm{x}$

58. Cui L, Zhou H, Zhao H, Zhou Y, Xu R, Xu X, et al. MicroRNA99a induces G1-phase cell cycle arrest and suppresses tumorigenicity in renal cell carcinoma. BMC Cancer. (2012) 12:546. doi: 10.1186/1471-2407$12-546$
59. Li W, Wang J, Chen QD, Qian X, Li Q, Yin Y, et al. Insulin promotes glucose consumption via regulation of miR-99a/mTOR/PKM2 pathway. PLoS ONE. (2013) 8:e64924. doi: 10.1371/journal.pone.0064924

60. Martínez-Fernández M, Paramio JM, Dueñas M. RNA detection in urine: from RNA extraction to good normalizer molecules. J Mol Diagnostics. (2016) 18:15-22. doi: 10.1016/j.jmoldx.2015.07.008

61. Armstrong DA, Dessaint JA, Ringelberg CS, Hazlett HF, Howard L, Abdalla MAK, et al. Pre-analytical handling conditions and small RNA recovery from urine for miRNA profiling. J Mol Diagnostics. (2018) 20:56571. doi: 10.1016/j.jmoldx.2018.04.003

62. Wachalska M, Koppers-Lalic D, van Eijndhoven M, Pegtel M, Geldof AA, Lipinska AD, et al. Protein complexes in urine interfere with extracellular vesicle biomarker studies. J Circ Biomarkers. (2016) 5:4. doi: 10.5772/62579

Conflict of Interest: The authors declare that the research was conducted in the absence of any commercial or financial relationships that could be construed as a potential conflict of interest.

Copyright $\odot 2021$ Hebbar, Chandel, Rani, Onteru and Singh. This is an open-access article distributed under the terms of the Creative Commons Attribution License (CC $B Y)$. The use, distribution or reproduction in other forums is permitted, provided the original author(s) and the copyright owner(s) are credited and that the original publication in this journal is cited, in accordance with accepted academic practice. No use, distribution or reproduction is permitted which does not comply with these terms. 